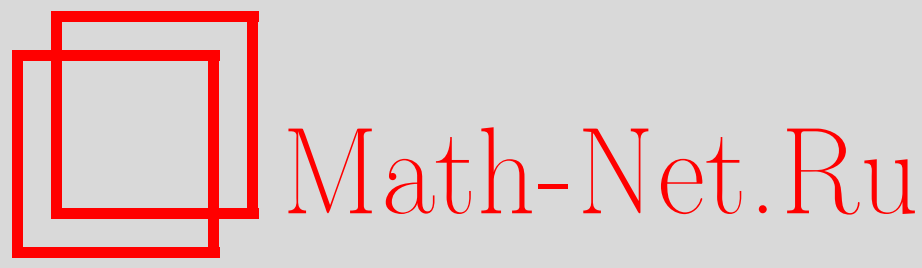

О. В. Ильин, Симметрии, функция тока и точные решения для двумерной стационарной кинетической модели Бродуэлла, ТМФ, 2014, том 179, номер 3, 350-359

DOI: https://doi.org/10.4213/tmf8641

Использование Общероссийского математического портала Math-Net.Ru подразумевает, что вы прочитали и согласны с пользовательским соглашением http://www . mathnet.ru/rus/agreement

Параметры загрузки:

IP: 3.95 .254 .165

26 апреля 2023 г., 06:47:10

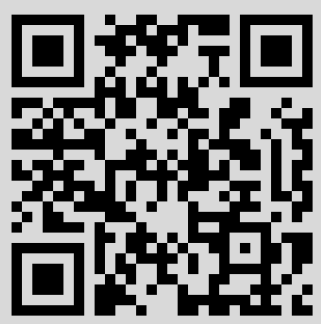




\section{СИММЕТРИИ, ФУНКЦИЯ ТОКА И ТОЧНЫЕ РЕШЕНИЯ ДЛЯ ДВУМЕРНОЙ СТАЦИОНАРНОЙ КИНЕТИЧЕСКОЙ МОДЕЛИ БРОДУЭЛЛА}

Стационарная кинетическая модель Бродуэлла для четырех скоростей на плоскости исследуется с помощью функции тока, которая удовлетворяет дифференциальному уравнению в частных производных. Для данного уравнения вычисляются алгебры классических и неклассических симметрий, затем строятся инвариантные решения. Все классы решений описывают непотенциальные течения. Рассматривается взаимосвязь между неклассическими симметриями и решениями, полученными ранее.

Ключевые слова: кинетическая модель Бродуэлла, функция тока, симметрии.

DOI: $10.4213 / \operatorname{tmf} 8641$

\section{1. ВВЕДЕНИЕ}

Система Бродуэлла для четырех скоростей является простейшей моделью, которая правильно передает многие свойства полного уравнения Больцмана для двух пространственных координат. Например, для системы Бродуэлла выполняются законы сохранения массы, импульса и энергии, верна $H$-теорема [1]. В стационарной форме модель имеет следующий вид:

$$
\frac{\partial f_{1}}{\partial x}=-\frac{\partial f_{2}}{\partial x}=-\frac{\partial f_{3}}{\partial y}=\frac{\partial f_{4}}{\partial y}=I(f), \quad I(f)=f_{3} f_{4}-f_{1} f_{2},
$$

где $f_{i}(x, y), i=1, \ldots, 4$, суть плотности частиц, движущихся в направлениях $\pm x$ и $\pm y$. Для построения решений этой модели в явном виде использовались различные методы. Все решения, отвечающие потенциальным течениям, были полностью описаны в работах [2]-[4]. В статье [5] два новых класса решений стационарной модели Бродуэлла были построены с помощью метода усеченного ряда Пенлеве (для нестационарной системы Карлемана основанные на данном методе решения, не являющиеся знакопостояннными, приведены в работе [6]), решения в автомодельной форме (для переменной, инвариантной относительно группы растяжений) получены

* Вычислительный центр им. А. А. Дородницына РАН, Москва, Россия. E-mail: oilyin@gmail.com 
в работе [7]. С помощью соответствия между решениями нестационарной системы Карлемана и стационарной моделью Бродуэлла в работах [4], [8] выведены решения, относящиеся к несжимаемому течению.

Из уравнений (1) получаем, что выполняются равенства

$$
f_{2}=-f_{1}+Q(y), \quad f_{4}=-f_{3}+S(x),
$$

где $Q(y)$ и $S(x)$ - неизвестные гладкие функции. С помощью этих равенств система (1) записывается в виде

$$
\frac{\partial f_{1}}{\partial x}=-\frac{\partial f_{3}}{\partial y}=f_{1}^{2}-f_{3}^{2}-Q(y) f_{1}+S(x) f_{3} .
$$

Введем функцию $\psi(x, y)$ по следующему правилу:

$$
f_{1}=\psi_{y}, \quad f_{3}=-\psi_{x}
$$

Тогда условие $\left(f_{1}\right)_{x}+\left(f_{3}\right)_{y}=0$ (вытекающее из уравнений $\left.(2)\right)$ выполняется как тождество, и любое из равенств в системе $(2)$ сводится к уравнению

$$
\psi_{x y}=\psi_{y}^{2}-\psi_{x}^{2}-S(x) \psi_{x}-Q(y) \psi_{y} .
$$

Итак, модель Бродуэлла редуцируется к уравнению (4), которое является предметом исследований настоящей статьи.

\section{2. МАКРОСКОПИЧЕСКИЕ ВЕЛИЧИНЫ, ФУНКЦИЯ ТОКА И ЗАВИХРЕННОСТЬ}

Определим плотность $\rho(x, y)$ и скорости потока $U(x, y), V(x, y)$ в направлениях $x, y$ с помощью следующих равенств:

$$
\begin{gathered}
\rho=f_{1}+f_{2}+f_{3}+f_{4}=P(y)+S(x), \\
\rho U=f_{1}-f_{2}=2 f_{1}-P(y), \quad \rho V=f_{3}-f_{4}=2 f_{3}-Q(x) .
\end{gathered}
$$

Очевидно, что выполняется уравнение непрерывности $(\rho U)_{x}+(\rho V)_{y}=0$. Это позволяет ввести функцию тока $\Psi(x, y)$ по правилу

$$
\Psi(x, y)=2 \psi(x, y)+\int^{x} Q(z) d z-\int^{y} P(z) d z
$$

Сразу получаем уравнения

$$
\rho U=\Psi_{y}=2 \psi_{y}-P, \quad \rho V=-\Psi_{x}=-2 \psi_{x}-Q(x),
$$

где функция $\psi$ задается формулами (3). Теперь определим скалярную завихренность $v$ :

$$
v=(\rho V)_{x}-(\rho U)_{y}=2\left(f_{3}\right)_{x}-2\left(f_{1}\right)_{y}+P_{y}-Q_{x} .
$$

Тогда скалярная завихренность течения запишется как $v=-2 \nabla^{2} \psi+P_{y}-Q_{x}$. 


\section{3. СИММЕТРИИ УРАВНЕНИЯ}

Для диференциальных уравнений с одной зависимой переменной (в нашем случае переменной $\psi$ ) векторное поле классических симметрий [9]-[12] представляется в следующем виде [12]:

$$
\begin{aligned}
X= & -F_{\psi_{x}} \partial_{x}-F_{\psi_{y}} \partial_{y}+\left(F-\psi_{x} F_{\psi_{x}}-\psi_{y} F_{\psi_{y}}\right) \partial_{\psi}+ \\
& +\left(F_{x}+F_{\psi} \psi_{x}\right) \partial_{\psi_{x}}+\left(F_{y}+F_{\psi} \psi_{y}\right) \partial_{\psi_{y}}
\end{aligned}
$$

где $F=F\left(x, y, \psi, \psi_{x}, \psi_{y}\right)$ - производящая функция.

Уравнение (4) содержит производные второго порядка, следовательно, требуется определить действие векторного поля симметрий на члены, содержащие такие производные. Это осуществляется с помощью формулы продолжения (см. монографию [12]), применяя которую, получаем поле $X_{2}$, действующее в пространстве переменных $\left(x, y, \psi, \psi_{x}, \psi_{y}, \psi_{x y}, \psi_{y y}, \psi_{x x}\right)$ :

$$
\begin{aligned}
X_{2}= & X+\left(D_{y}\left(F_{x}+F_{\psi} \psi_{x}\right)+\psi_{x x} D_{y}\left(F_{\psi_{x}}\right)+\psi_{x y} D_{y}\left(F_{\psi_{y}}\right)\right) \partial_{\psi_{x y}}+ \\
& +\left(D_{x}\left(F_{x}+F_{\psi} \psi_{x}\right)+\psi_{x x} D_{x}\left(F_{\psi_{x}}\right)+\psi_{x y} D_{x}\left(F_{\psi_{y}}\right)\right) \partial_{\psi_{x x}}+ \\
& +\left(D_{y}\left(F_{y}+F_{\psi} \psi_{y}\right)+\psi_{x y} D_{y}\left(F_{\psi_{x}}\right)+\psi_{y y} D_{y}\left(F_{\psi_{y}}\right)\right) \partial_{\psi_{y y}},
\end{aligned}
$$

где $D_{x}, D_{y}$ - полные производные, задаваемые соотношениями

$$
\begin{aligned}
& D_{x}=\partial_{x}+\psi_{x} \partial_{\psi}+\psi_{x x} \partial_{\psi_{x}}+\psi_{x y} \partial_{\psi_{y}}+\cdots, \\
& D_{y}=\partial_{y}+\psi_{y} \partial_{\psi}+\psi_{y y} \partial_{\psi_{y}}+\psi_{x y} \partial_{\psi_{x}}+\cdots
\end{aligned}
$$

Векторное поле (6) является симметрией уравнения (4), если выполнено условие

$$
\left.X_{2}(E)\right|_{E=0}=0, \quad E \equiv-\psi_{x y}+\psi_{y}^{2}-\psi_{x}^{2}-S(x) \psi_{x}-Q(y) \psi_{y} .
$$

Раскрывая это условие, получаем следующее уравнение относительно функции $F$ :

$$
\begin{aligned}
& \left(F_{\psi_{x}} S_{x} \psi_{x}+F_{\psi_{y}} Q_{y} \psi_{y}-\left(2 \psi_{x}+S\right)\left(F_{x}+F_{\psi} \psi_{x}\right)+\left(2 \psi_{y}-Q\right)\left(F_{y}+F_{\psi} \psi_{y}\right)-\right. \\
& \quad-\left\{F_{x y}+F_{x \psi} \psi_{y}+F_{x \psi_{x}} \psi_{x y}+F_{x \psi_{y}} \psi_{y y}+F_{\psi} \psi_{x y}+F_{y \psi} \psi_{y}+F_{\psi \psi} \psi_{x} \psi_{y}+\right. \\
& \quad+F_{\psi \psi_{x}} \psi_{x} \psi_{x y}+F_{\psi \psi_{y}} \psi_{x} \psi_{y y}+\left(F_{y \psi_{x}}+F_{\psi \psi_{x}} \psi_{y}+F_{\psi_{x} \psi_{x}} \psi_{x y}+F_{\psi_{x}} \psi_{y} \psi_{y y}\right) \psi_{x x}+ \\
& \left.\left.\quad+\left(F_{y \psi_{y}}+F_{\psi \psi_{y}} \psi_{y}+F_{\psi_{x} \psi_{y}} \psi_{x y}+F_{\psi_{y}} \psi_{y} \psi_{y y}\right) \psi_{x y}\right\}\right)\left.\right|_{E=0}=0
\end{aligned}
$$

Соберем все коэффициенты при $\psi_{x x} \psi_{y y}, \psi_{x x}, \psi_{y y}$ и получим уравнения

$$
\begin{aligned}
\psi_{x x} \psi_{y y}: & F_{\psi_{x} \psi_{y}}=0 \\
\psi_{x x}: & F_{y \psi_{x}}+F_{\psi \psi_{x}} \psi_{y}+F_{\psi_{x} \psi_{x}} \psi_{x y}=0, \\
\psi_{y y}: & F_{x \psi_{y}}+F_{\psi \psi_{y}} \psi_{x}+F_{\psi_{y} \psi_{y}} \psi_{x y}=0 .
\end{aligned}
$$

Из этих уравнений заключаем, что функция $F$ линейна по переменным $\psi_{x}, \psi_{y}$ :

$$
F=a(x) \psi_{x}+b(y) \psi_{y}+c(x, y, \psi) .
$$

Приравнивая к нулю коэффициенты при $\psi_{x} \psi_{y}$, получаем уравнение $F_{\psi \psi}=0$, тогда

$$
F=a(x) \psi_{x}+b(y) \psi_{y}+c(x, y) \psi+d(x, y),
$$


где $a(x), b(y), c(x, y), d(x, y)$ - некоторые неизвестные функции. Подставляем равенство (8) в уравнение (7) и вместо (7) получаем

$$
\begin{aligned}
a S_{x} \psi_{x} & +b Q_{y} \psi_{y}-\left(2 \psi_{x}+S\right)\left(a_{x} \psi_{x}+c_{x} \psi+d_{x}+c \psi_{x}\right)+ \\
& +\left(2 \psi_{y}-Q\right)\left(b_{y} \psi_{y}+c_{y} \psi+d_{y}+c \psi_{y}\right)-\left(c_{x y} \psi+d_{x y}+c_{x} \psi_{y}+c_{y} \psi_{y}\right)- \\
& -\left(\psi_{y}^{2}-\psi_{x}^{2}-S(x) \psi_{x}-Q(y) \psi_{y}\right)\left(a_{x}+c+b_{y}\right)=0 .
\end{aligned}
$$

Рассмотрим слагаемые, содержащие $\psi$ :

$$
-\left(2 \psi_{x}+S\right) c_{x}+\left(2 \psi_{y}-Q\right) c_{y}-c_{x y}=0 .
$$

Из этого уравнения следует, что $c_{x}=c_{y}=0$.

Теперь соберем члены при $\psi_{x}^{2}$ и $\psi_{y}^{2}$, получим

$$
\begin{array}{lrl}
\psi_{x} \psi_{x}: & -2 a_{x}-2 c+\left(a_{x}+b_{y}+c\right)=0, \\
\psi_{y} \psi_{y}: & 2 b_{y}+2 c-\left(a_{x}+b_{y}+c\right)=0 .
\end{array}
$$

Решаем эти уравнения и получаем, что $c=0$ и $a_{x}=b_{y}=K$, где $K$ - постоянная. Тогда

$$
a=K x+\alpha, \quad b=K y+\beta,
$$

где $\alpha, \beta$ - постоянные.

Рассмотрим коэффициенты при $\psi_{x}, \psi_{y}$ :

$$
\begin{array}{ll}
\psi_{x}: & a S_{x}-2 d_{x}+K S=0, \\
\psi_{y}: & b Q_{y}+2 d_{y}+K Q=0 .
\end{array}
$$

Теперь соберем все члены, не содержащие функции $\psi$ и ее производных, получим уравнение

$$
S d_{x}+Q d_{y}+d_{x y}=0 .
$$

Продифференцируем уравнение (9) по $y$ (или уравнение (10) по $x$ ), получим $d_{x y}=0$. Отсюда имеем

$$
d(x, y)=d_{1}(x)+d_{2}(y)
$$

где $d_{1}(x), d_{2}(y)$ - неизвестные функции. Итак,

$$
F=(K x+\alpha) \psi_{x}+(K y+\beta) \psi_{y}+d_{1}(x)+d_{2}(y) .
$$

Уравнение (11) сводится к уравнению

$$
S d_{x}+Q d_{y}=0
$$

Теперь, вспоминая, что $S d_{x}$ есть функция от переменной $x$, а $Q d_{y}$ есть функция от переменной $y$, из уравнения (12) выводим, что выполняются следующие равенства:

$$
S d_{x}=L, \quad Q d_{y}=-L,
$$

где $L$ - постоянная. Теперь подставим выражения для $d_{x}$ и $d_{y}$ из равенств $(13)$ в уравнения (9), (10):

$$
\begin{aligned}
& \psi_{x}: \quad(K x+\alpha) S_{x}-2 L S^{-1}+K S=0, \\
& \psi_{y}: \quad(K y+\beta) Q_{y}-2 L Q^{-1}+K Q=0 .
\end{aligned}
$$


Следует рассмотреть два случая. Если постоянная $K \neq 0$, тогда решения уравнений (14) имеют следующий вид:

$$
S(x)=\sqrt{\frac{C_{1}}{(K x+\alpha)^{2}}+M}, \quad Q(y)=\sqrt{\frac{C_{2}}{(K y+\beta)^{2}}+M},
$$

где $C_{1}, C_{2}, \alpha, \beta, M$ - постоянные. Производящая функция $F$ такова:

$$
F=(K x+\alpha) \psi_{x}+(K y+\beta) \psi_{y}+\frac{1}{2} \sqrt{M(K x+\alpha)^{2}+C_{1}}-\frac{1}{2} \sqrt{M(K y+\beta)^{2}+C_{2}}+\lambda .
$$

В случае $K=0$ решения уравнений (14) записываются как

$$
S(x)=\sqrt{\frac{4 L}{\alpha} x+C_{1}}, \quad Q(y)=\sqrt{\frac{4 L}{\beta} y+C_{2}},
$$

где $\alpha \neq 0, \beta \neq 0$ - постоянные. Производящая функция имеет вид

$$
F=\alpha \psi_{x}+\beta \psi_{y}+\frac{\alpha}{2} \sqrt{\frac{4 L}{\alpha} x+C_{1}}-\frac{\beta}{2} \sqrt{\frac{4 L}{\beta} y+C_{2}}+\lambda .
$$

Сформулируем полученные результаты в виде утверждения.

УтвеРЖДЕНИЕ 1. Рассмотрим уравнение (4)

$$
\psi_{x y}=\psi_{y}^{2}-\psi_{x}^{2}-S(x) \psi_{x}-Q(y) \psi_{y}
$$

Eсли

$$
S(x)=\sqrt{\frac{C_{1}}{(K x+\alpha)^{2}}+M}, \quad Q(y)=\sqrt{\frac{C_{2}}{(K y+\beta)^{2}}+M},
$$

то классические симметрии уравнения (4) задаются полем

$$
\begin{aligned}
X= & -(K x+\alpha) \partial_{x}-(K y+\beta) \partial_{y}+ \\
& +\left(\frac{1}{2} \sqrt{M(K x+\alpha)^{2}+C_{1}}-\frac{1}{2} \sqrt{M(K y+\beta)^{2}+C_{2}}+\lambda\right) \partial_{\psi},
\end{aligned}
$$

где $K \neq 0, C_{1}, C_{2}, \alpha, \beta, M, \lambda$ - постоянные. $B$ случае

$$
S(x)=\sqrt{\frac{L}{\alpha} x+C_{1}}, \quad Q(y)=\sqrt{\frac{L}{\beta} y+C_{2}}
$$

классические симметрии уравнения (4) задаются полем

$$
X=-\alpha \partial_{x}-\beta \partial_{y}+\left(\frac{\alpha}{2} \sqrt{\frac{L}{\alpha} x+C_{1}}-\frac{\beta}{2} \sqrt{\frac{L}{\beta} y+C_{2}}+\lambda\right) \partial_{\psi},
$$

где $\alpha \neq 0, \beta \neq 0, C_{1}, C_{2}, L, \lambda$ - постоянные. 


\section{4. ИНВАРИАНТНЫЕ РЕШЕНИЯ СИСТЕМЫ БРОДУЭЛЛА}

В качестве приложения результатов утверждения 1 построим инвариантные относительно группы симметрий решения уравнения (4) и, как следствие, модели Бродуэлла. Очевидно, что физический смысл имеют только неотрицательные плотности. Для всех решений, построенных в данном разделе, существуют ограниченные прямоугольные области $x \in\left[a_{1}, a_{2}\right], y \in\left[b_{1}, b_{2}\right]$, в которых функции $f_{j}, j=1, \ldots, 4$, неотрицательны и удовлетворяют граничным условиям

$$
f_{1}\left(a_{1}, y\right)=F_{1}(y), \quad f_{2}\left(a_{2}, y\right)=F_{2}(y), \quad f_{3}\left(x, b_{1}\right)=F_{3}(x), \quad f_{4}\left(x, b_{2}\right)=F_{4}(x),
$$

где $F_{j}, j=1, \ldots, 4,-$ плотности частиц на границах. В работе [13] показано, что данная задача разрешима в прямоугольнике, если граничные плотности суть непрерывные функции. С физической точки зрения условия (17) определяют испарение газа с границ прямоугольной области.

СЛУЧАЙ 1. Пусть

$$
Q(x)=\sqrt{\frac{C_{1}}{(K x+\alpha)^{2}}+M}, \quad P(y)=\sqrt{\frac{C_{2}}{(K y+\beta)^{2}}+M}, \quad K \neq 0 .
$$

Для простоты положим $\lambda=0$. Поле (15) обладает следующими инвариантами:

$$
I_{1}=\frac{K y+\beta}{K x+\alpha}, \quad I_{2}=-\psi+g_{1}(x)+g_{2}(y),
$$

где

$$
\left(g_{1}\right)_{x}=-\frac{1}{2} Q(x), \quad\left(g_{2}\right)_{y}=\frac{1}{2} P(y) .
$$

Следовательно, решения следует искать в форме

$$
\psi=f(\xi)+g_{1}(x)+g_{2}(y), \quad \xi \equiv \frac{K y+\beta}{K x+\alpha} .
$$

Подставим эти выражения в уравнение (4), получим следующее обыкновенное дифференциальное уравнение:

$$
-f_{\xi}-\xi f_{\xi \xi}=f_{\xi}^{2}-\xi^{2} f_{\xi}^{2}+\left(4 K^{2}\right)^{-1} C_{1}-\left(4 K^{2}\right)^{-1} C_{2} \xi^{-2}
$$

Оно сводится к уравнению типа Рикатти подстановкой $f(\xi)_{\xi}=\xi^{-1} h(\xi)$, где $h(\xi)-$ неизвестная функция:

$$
h_{\xi}+\left(1-\xi^{2}\right) \xi^{-2} h^{2}+\left(4 K^{2}\right)^{-1}\left(C_{1}-C_{2} \xi^{-2}\right)=0 .
$$

Рассмотрим случай $C_{1}=C_{2}=C>0$. Тогда уравнение (18) имеет следующее решение:

$$
h(\xi)=\left[B \exp \left(-\frac{\sqrt{C}}{K}\left(\xi^{-1}+\xi\right)\right)-\frac{K}{\sqrt{C}}\right]^{-1}+\frac{\sqrt{C}}{2 K},
$$

где $B$ - постоянная интегрирования. Тогда

$$
\psi=g_{1}(x)+g_{2}(y)+\int^{\frac{K y+\beta}{K x+\alpha}} \frac{d \eta}{\eta}\left(\left[B \exp \left(-\frac{\sqrt{C}}{K}\left(\xi^{-1}+\xi\right)\right)-\frac{K}{\sqrt{C}}\right]^{-1}+\frac{\sqrt{C}}{2 K}\right) .
$$


Используя равенства $f_{1}=\psi_{y}, f_{2}=-\psi_{y}+Q, f_{3}=-\psi_{x}, f_{4}=\psi+S$, получаем решения модели Бродуэлла (1):

$$
\begin{aligned}
f_{1,2}= & \pm \frac{K}{K y+\beta}\left[B \exp \left(-\frac{\sqrt{C}}{K}\left(\xi^{-1}+\xi\right)\right)-\frac{K}{\sqrt{C}}\right]^{-1} \pm \\
& \pm \frac{\sqrt{C}}{2(K y+\beta)}+\frac{1}{2} \sqrt{\frac{C}{(K y+\beta)^{2}}+M,} \\
f_{3,4}= & \pm \frac{K}{K x+\alpha}\left[B \exp \left(-\frac{\sqrt{C}}{K}\left(\xi^{-1}+\xi\right)\right)-\frac{K}{\sqrt{C}}\right]^{-1} \pm \\
& \pm \frac{\sqrt{C}}{2(K x+\beta)}+\frac{1}{2} \sqrt{\frac{C}{(K x+\beta)^{2}}+M,}
\end{aligned}
$$

где $C>0, K \neq 0, M, \alpha, \beta, B$ - постоянные. Очевидно, что только неотрицательные плотности $f_{j}, j=1, \ldots, 4$, имеют физический смысл. Следовательно, необходимо определить области на плоскости $(x, y)$, в которых $f_{j}(x, y) \geqslant 0, j=1, \ldots, 4$.

Рассмотрим прямоугольник $\left[x_{0}, x_{1}\right] \times\left[y_{0}, y_{1}\right]$, пусть $x_{1}>x_{0}>0, y_{1}>y_{0}>0$ и величины $C>0, K>0, \alpha>0, \beta>0$ произвольны. Возьмем $B>K / \sqrt{C}$. Все члены при \pm в (19) ограничены в рассматриваемом прямоугольнике. Тогда, выбирая постоянную $M$ достаточно большой, получаем, что $f_{j}(x, y) \geqslant 0, j=1, \ldots, 4$, в области $\left[x_{0}, x_{1}\right] \times\left[y_{0}, y_{1}\right]$.

В частном случае $\alpha=\beta=M=0, K=1$ функции (19) переходят в решения, лежащие в одном из классов, найденных в работе [7].

Явное выражение для завихренности (5) в данном случае громоздко, но можно убедиться, что оно не равно нулю.

СЛУчАЙ 2. Пусть

$$
Q(x)=\sqrt{\frac{L}{\alpha} x+C_{1}}, \quad P(y)=\sqrt{\frac{L}{\beta} y+C_{2}}, \quad \alpha, \beta \neq 0 .
$$

Инварианты поля (16) равны

$$
I_{1}=\beta x-\alpha y, \quad I_{2}=-\psi+g_{1}(x)+g_{2}(y),
$$

где

$$
\left(g_{1}\right)_{x}=-\frac{1}{2} Q(x)+\frac{\lambda}{2}, \quad\left(g_{2}\right)_{y}=\frac{1}{2} P(y)+\frac{\lambda}{2} .
$$

Тогда можно построить решения уравнения (4) в следующем виде:

$$
\psi=f(z)+g_{1}(x)+g_{2}(y), \quad z \equiv \beta x-\alpha y,
$$

где $f(z)$ - неизвестная функция. Как и в предыдущем случае, получаем обыкновенное дифференциальное уравнение второго порядка относительно функции $f(z)$ :

$$
\alpha \beta f_{z z}-\lambda(\alpha+\beta) f_{z}+\left(\alpha^{2}-\beta^{2}\right) f_{z}^{2}+\left(\frac{L}{4 \alpha \beta}\right) z+\frac{1}{4}\left(C_{1}-C_{2}\right)=0 .
$$


Построим решения этого уравнения в трех случаях: при $\alpha=-1, \beta=1$, при $\lambda=0$, $\alpha=1, \beta=1$ и при $\lambda \neq 0, \alpha=1, \beta=1$.

Если $\alpha=-1, \beta=1$, то

$$
\psi=g_{1}(x)+g_{2}(y)-\frac{L}{24}(x+y)^{3}+\frac{1}{8}\left(C_{1}-C_{2}\right)(x+y)^{2}+K(x+y) .
$$

Соответствующие решения модели Бродуэлла записываются в следующем виде:

$$
\begin{aligned}
& f_{1,2}=\mp \frac{L}{8}(x+y)^{2} \pm \frac{1}{4}\left(C_{1}-C_{2}\right)(x+y) \pm\left(K+\frac{\lambda}{2}\right)+\frac{1}{2} \sqrt{L y+C_{2}}, \\
& f_{3,4}= \pm \frac{L}{8}(x+y)^{2} \mp \frac{1}{4}\left(C_{1}-C_{2}\right)(x+y) \mp\left(K+\frac{\lambda}{2}\right)+\frac{1}{2} \sqrt{-L x+C_{1}},
\end{aligned}
$$

где $L, C_{1}, C_{2}, K, \lambda$ - постоянные. Найдем области на плоскости, в которых функции $f_{j}, j=1, \ldots, 4$, неотрицательны. Для простоты положим $C_{1}=C_{2}=C>0$. Как и в предыдущем случае, рассмотрим прямоугольник $\left[x_{0}, x_{1}\right] \times\left[y_{0}, y_{1}\right]$ при $x_{1}>x_{0}>0$, $y_{1}>y_{0}>0$. Все слагаемые при,$\pm \mp$ в выражениях для $f_{i}$ ограничены в рассматриваемой области. Выбирая постоянную $C>0$ достаточно большой, получаем, что функции $f_{j}, j=1, \ldots, 4$, неотрицательны в прямоугольнике.

Завихренность вычисляется по формуле (5):

$$
v=L(x+y)-\left(C_{1}-C_{2}\right)
$$

т. е. течение непотенциально.

Далее рассмотрим случай $\lambda=0, \alpha=\beta=1$. Решая уравнение (20), получаем выражение для функции $\psi$ :

$$
\psi=g_{1}(x)+g_{2}(y)-\frac{L}{24}(x-y)^{3}-\frac{1}{8}\left(C_{1}-C_{2}\right)(x-y)^{2}+K(x-y) .
$$

В итоге получаем решения модели Бродуэлла:

$$
\begin{aligned}
& f_{1,2}= \pm \frac{L}{8}(x-y)^{2} \pm \frac{1}{4}\left(C_{1}-C_{2}\right)(x-y) \mp K+\frac{1}{2} \sqrt{L y+C_{2}}, \\
& f_{3,4}= \pm \frac{L}{8}(x-y)^{2} \pm \frac{1}{4}\left(C_{1}-C_{2}\right)(x-y) \mp K+\frac{1}{2} \sqrt{L x+C_{1}}
\end{aligned}
$$

где $L, C_{1}, C_{2}, K$ - постоянные. Аналогично предыдущему случаю получаем, что функции $f_{j}, j=1, \ldots, 4$, неотрицательны в той же области $(x, y) \in\left[x_{0}, x_{1}\right] \times\left[y_{0}, y_{1}\right]$, где $x_{1}>x_{0}>0, y_{1}>y_{0}>0$, если выбрать достаточно большие значения постоянных $C_{1}=C_{2}=C>0$.

Завихренность решений снова отлична от нуля:

$$
v=L(x-y)+\left(C_{1}-C_{2}\right) .
$$

Теперь в уравнении (20) положим $\lambda \neq 0, \alpha=\beta=1$, получаем в итоге выражение для функции $\psi$ :

$$
\psi=g_{1}(x)+g_{2}(y)+\psi_{0} e^{2 \lambda(x-y)}+\left(\frac{L}{16 \lambda}\right)(x-y)^{2}+\frac{1}{8 \lambda}\left(\frac{L}{2 \lambda}+\left(C_{1}-C_{2}\right)\right)(x-y) .
$$


Используя формулы $f_{1}=\psi_{y}, f_{2}=-\psi_{y}+Q, f_{3}=-\psi_{x}, f_{4}=\psi+S$, получаем решения модели Бродуэлла

$$
\begin{aligned}
& f_{1,2}=\mp 2 \lambda \psi_{0} e^{2 \lambda(x-y)} \mp\left(\frac{L}{8 \lambda}\right)(x-y) \mp \frac{1}{8 \lambda}\left(\frac{L}{2 \lambda}+\left(C_{1}-C_{2}\right)\right) \pm \frac{\lambda}{2}+\frac{1}{2} \sqrt{L y+C_{2}}, \\
& f_{3,4}=\mp 2 \lambda \psi_{0} e^{2 \lambda(x-y)} \mp\left(\frac{L}{8 \lambda}\right)(x-y) \mp \frac{1}{8 \lambda}\left(\frac{L}{2 \lambda}+\left(C_{1}-C_{2}\right)\right) \mp \frac{\lambda}{2}+\frac{1}{2} \sqrt{L x+C_{1}},
\end{aligned}
$$

где $\lambda \neq 0, \psi_{0}, L, C_{1}, C_{2}$ - постоянные. Аналогично предыдущим случаям, если $C_{1}=C_{2}$, то в любом прямоугольнике $\left[x_{0}, x_{1}\right] \times\left[y_{0}, y_{1}\right]$, где $x_{1}>x_{0}>0, y_{1}>y_{0}>0$, можно добиться неотрицательности плотностей $f_{j}(x, y), j=1, \ldots, 4$, выбирая постоянную $C_{1}=C_{2}$ достаточно большой.

Завихренность решений отлична от нуля:

$$
v=-16 \lambda^{2} \psi_{0} e^{2 \lambda(x-y)}-\frac{L}{2 \lambda} \neq 0
$$

\section{5. НЕКЛАССИЧЕСКИЕ СИММЕТРИИ И РЕШЕНИЯ МОДЕЛИ БРОДУЭЛЛА}

Существуют решения модели Бродуэлла, которые невозможно получить с помощью симметрий, вычисленных в предыдущих разделах. Например, рассмотрим одно из решений, полученных в работе [5] с помощью метода усеченного ряда Пенлеве:

$$
\begin{array}{ll}
f_{1}=-\frac{c_{1} x}{L x y+\left(x+c_{1} y\right)\left(c_{1} x+y\right)}, & f_{2}=-f_{1}+a y, \\
f_{3}=-\frac{c_{1} y}{L x y+\left(x+c_{1} y\right)\left(c_{1} x+y\right)}, & f_{4}=-f_{3}+a x,
\end{array}
$$

где $a, L, c_{1} \neq \pm 1$ - постоянные. Очевидно, что утверждение 1 не охватывает решения (21). Тем не менее их можно получить с помощью неклассических симметрий.

Рассмотрим векторное поле $Y=x \partial_{x}+y \partial_{y}$. Производящая функция этого поля есть $F=-x \psi_{x}-y \psi_{y}$. Второе продолжение для поля $Y$ имеет следующий вид:

$$
Y_{2}=Y-\psi_{x} \partial_{\psi_{x}}-\psi_{y} \partial_{\psi_{y}}-2 \psi_{x y} \partial_{\psi_{x y}}
$$

Рассмотрим уравнение (4) при условии, что $S(x)=a x, Q(y)=a y, a \neq 0$. Имеем

$$
\left.Y_{2}(E)\right|_{E=0}=-2 a\left(x \psi_{x}+y \psi_{y}\right)=2 a F \neq 0,
$$

где

$$
E \equiv-\psi_{x y}+\psi_{y}^{2}-\psi_{x}^{2}-S(x) \psi_{x}-Q(y) \psi_{y},
$$

следовательно, поле $Y$ не является классической симметрией. Однако поле $Y$ является частной симметрией [14]-[16] уравнения (4), а именно условие $Y_{2}(E)=0$ выполняется на пересечении многообразий $E=0$ и $E_{F}=0$, где $E_{F}$ задается равенством $F\left(x, y, \psi, \psi^{(1)}\right)=0$ :

$$
\left.Y_{2}(E)\right|_{E \cap E_{F}}=0 .
$$

Очевидно, решения уравнения (4), инвариантные относительно поля $Y=x \partial_{x}+y \partial_{y}$, следует искать в виде $\psi=f(z)$, где $z \equiv y / x$. В итоге получаются равенства $(21)$. 
Следует отметить,что нахождение неклассических симметрий представляется перспективным инструментом для поиска решений других кинетических моделей.

В заключение отметим еще один интересный момент. Уравнение (4) в случае $S(x)=a x, Q(y)=a y, a \neq 0$ допускает более общую неклассическую симметрию

$$
x \partial_{x}+y \partial_{y}+w \partial_{\psi}, \quad w \in \mathbb{R} .
$$

Решения, инвариантные относительно этого поля, имеют вид $\psi=w \ln r+f(z)$, где $r \equiv\left(x^{2}+y^{2}\right)^{1 / 2}, z \equiv y / x$. Подстановка данного анзаца в уравнение (4) приводит к обыкновенному дифференциальному уравнению типа Риккати относительно функции $f(z)$, которое, по-видимому, имеет решение в элементарных функциях только в случае $w=0$. Тем не менее рассматриваемый функциональный вид решения $\psi=w \ln r+f(z)$ представляет интерес в силу следующих обстоятельств. Во-первых, подстановка $\psi=w \ln r+f(z)$ является примером анзаца Кларксона-Крускала [17], позволяющего свести уравнение в частных производных к обыкновенному дифференциальному уравнению. Связь между методом Кларксона-Крускала и неклассическими симметриями обсуждается в монографии [15]. Во-вторых, нетрудно видеть, что подстановка $\psi=w \ln r+f(z)$ представляет собой решение в полярных координатах с разделенными переменными. Вполне вероятно, что переход к полярным координатам (или сферическим в случае трехмерных моделей) позволит находить решения более сложных дискретных кинетических моделей.

\section{Список литературы}

[1] С. К. Годунов, У. М. Султангазин, УМН, 26:3(159) (1971), 3-51.

[2] A. V. Bobylev, G. Spiga, J. Phys. A, 27:22 (1994), 7451-7459.

[3] A. V. Bobylev, G. Caraffini, G. Spiga, Eur. J. Mech. B/Fluids, 19:2 (2000), 303-315.

[4] A. V. Bobylev, Math. Methods Appl. Sci., 19:10 (1996), 825-845.

[5] O. Ilyin, J. Stat. Phys., 146:1 (2012), 67-72.

[6] О. Линдблом, Н. Эйлер, ТМФ, 131:2 (2002), 179-193.

[7] О. В. Ильин, ТМФ, 170:3 (2012), 481-488.

[8] H. Cabannes, Eur. J. Mech. B/Fluids, 16:1 (1997), 1-15.

[9] V.V. Zharinov, Lecture Notes on Geometrical Aspects of Partial Differential Equations, Series on Soviet and East European Mathematics, 9, World Sci., Singapore, 1992.

[10] N.H. Ibragimov, Transformation Groups Applied to Mathematical Physics, Reidel, Dordrecht, 1985.

[11] П. Олвер, Приложения групп Ли к дифференииальным уравнениям, Мир, М., 1989.

[12] I. S. Krasil'shchik, P. H. M. Kersten, Symmetries and Recursion Operators for Classical and Supersymmetric Differential Equations, Mathematics and its Applications, 507, Kluwer, Dordrecht, 2000.

[13] C. Cercignani, R. Illner, M. Shinbrot, Commun. Math. Phys., 114:4 (1988), 687-698.

[14] P. J. Olver, Appl. Numer. Math., 10:3-4 (1992), 307-324.

[15] P. J. Olver, E. M. Vorob'ev, "Nonclassical and conditional symmetries", CRC Handbook of Lie Group Analysis of Differential Equations, v. 3: New Trends in Theoretical Developments and Computational Methods, ed. N. H. Ibragimov, CRC Press, Boca Raton, FL, 1996, 291-328.

[16] P. J. Olver, P. Rosenau, SIAM J. Appl. Math., 47:2 (1987), 263-278.

[17] P. A. Clarkson, M. D. Kruskal, J. Math. Phys., 30:10 (1989), 2201-2213. 